\title{
Molecular Detection of Toxoplasma gondii and Neospora caninum in Domestic Ducks in Hunan Province, China
}

\section{OPEN ACCESS}

Edited by: Annunziata Giangaspero, University of Foggia, Italy

Reviewed by:

Stefania Zanet,

University of Turin, Italy

Quan Liu,

Foshan University, China Alice Vismarra,

University of Parma, Italy

${ }^{*}$ Correspondence:

Guo-Hua Liu linguohua5202008@163.com

Specialty section: This article was submitted to Parasitology,

a section of the journal

Frontiers in Veterinary Science

Received: 05 January 2021 Accepted: 22 March 2021

Published: 15 April 2021

Citation:

$L v Q-Y$, Zheng $H-L$, Yang $W-H$ and Liu G-H (2021) Molecular Detection of

Toxoplasma gondii and Neospora caninum in Domestic Ducks in Hunan

Province, China.

Front. Vet. Sci. 8:649603. doi: 10.3389/fvets.2021.649603

\author{
Qiu-Yan Lv ${ }^{1}$, He-Liang Zheng ${ }^{1}$, Wen-He Yang ${ }^{1}$ and Guo-Hua Liu ${ }^{1,2 *}$ \\ ${ }^{1}$ Hunan Provincial Key Laboratory of Protein Engineering in Animal Vaccines, College of Veterinary Medicine, Hunan \\ Agricultural University, Changsha, China, ${ }^{2}$ Hunan Co-Innovation Center of Animal Production Safety, Changsha, China
}

Toxoplasma gondii and Neospora caninum are protozoan parasites that infect warm-blooded animals, and cause major economic losses in livestock industries worldwide. However, little is known about the genotypes of T. gondii and N. caninum in domestic ducks in China. Herein, brain samples from 588 domestic ducks from Hunan province in China were examined for the presence of $T$. gondii and $N$. caninum. Polymerase chain reaction (PCR) was used to detect $T$. gondii B1 gene and $N$. caninum NC-5 gene. Forty-five DNA samples (7.7\%; 95\% Cl: 5.5-9.9) were positive for B1 gene, and two (0.3\%; 95\% Cl: 0-0.7) were positive for NC-5 gene. The risk factors significantly associated with $T$. gondii infection were age and sex. The 45 samples positive for $T$. gondii were genotyped using multi-locus PCR-RFLP analysis and only one sample was fully genotyped as ToxoDB\#9 (Chinese I). These results provide new information about the epidemiology of T. gondii and N. caninum in ducks in Hunan province in China. The data also highlight the importance of a "One Health" approach to dealing with toxoplasmosis.

Keywords: Toxoplasma gondii, Neospora caninum, domestic ducks, PCR-RFLP, China

\section{INTRODUCTION}

Toxoplasma gondii and Neospora caninum are two important and highly prevalent protozoan parasites $(1,2)$. Toxoplasmosis, caused by $T$. gondii, is a widespread zoonotic disease causing significantly economic losses in animals and serious public health impacts on humans $(3,4)$. T. gondii in pregnant women may be transmitted to fetus and cause severe neurological sequelae $(5,6)$. Neosporosis, caused by N. caninum, is one of the most important causes of abortion in ruminants, particularly in cattle $(7,8)$. N caninum is not considered a zoonotic parasite, but low antibody titers to $N$. caninum have been reported in humans (9-11).

Domestic cats and wild felids serve as definitive hosts of T. gondii, while dogs and wild canines play the role of definitive hosts of $N$. caninum. Other warm-blooded vertebrate animals (including birds) have been reported as intermediated hosts for both T. gondii and N. caninum (12-14). Various avian species play an important role in the life cycle of these parasites by serving as intermediate hosts. Avian species can be infected by $T$. gondii and N. caninum mainly via ingestion of sporulated oocysts from contaminated environments by feline and canine feces, respectively $(15,16)$. Domestic ducks serve as a common food source particularly in China. The per capita consumption of duck meat in China was $6.75 \mathrm{~kg}$ in 2019. Chinese people often eat undercooked duck meat as roast, spicy or dried. Additionally, duck blood in chili sauce (undercooked food) has recently become popular in many parts of China. 
In China, ToxoDB\#9 (also named as Chinese I) is the most common genotype in domestic animals, followed by ToxoDB\#10 $(17,18)$. However, limited information is available concerning the molecular prevalence of T. gondii in domestic ducks in China. Only one study carried out by Zou et al. (19) showed that genotype ToxoDB\#9 was predominant in poultry (including 115 duck meats) in Shandong province of China, indicating that the genetic variation of T. gondii in poultry in this province is limited. In addition, low antibody titers to $N$. caninum were found and $N$. caninum DNA was detected in wild waterfowl in Italy, which suggests that wild waterfowl is susceptible to N. caninum (20). Nonetheless, domestic ducks as natural intermediate host of $N$. caninum have not been reported.

The aim of the present study was to determine the molecular prevalence, risk factors and genotypes of T. gondii and $N$. caninum in domestic ducks intended for human consumption in Hunan province, China. The results provide a baseline for future surveillance and control programs of these parasites in ducks in China.

\section{MATERIALS AND METHODS}

\section{Sample Collection}

From October 2018 to March 2020, 588 free-range ducks were purchased from food markets in five representative regions of Hunan province, China (Table 1). From each food market, 5\% of the slaughtered ducks were randomly sampled, where brain tissue was collected from each ducks and frozen at $-20^{\circ} \mathrm{C}$ until assayed. Information about the geographic region, season, sex, and age of each duck was gathered.

\section{DNA Extraction and PCR Amplification}

Approximately $30 \mathrm{mg}$ was obtained from each brain sample and total genomic DNA was extracted using a commercial kit (Wizard ${ }^{\circledR}$ SV Genomic DNA Purification System, Promega, Madison, USA) according to the manufacturer's directions. A semi-nested PCR was performed to detect T. gondii B1 gene (131 bp) as previously described (21). This gene target has been extensively used for detecting $T$. gondii infection in pigs, sheep, chicken, and other animals (22-25). Two primer pairs were used to amplify regions of the $\mathrm{B} 1$ gene of $T$. gondii: the outer primers B1-F1: 5'-GGAACTGCATCCGTTCATGAG$3^{\prime}$ and B1-R1: 5'-TCTTTAAAGCGTTCGTGGTC-3'; and inner primers B1-F2: 5'-TGCATAGGTTGCAGTCACTG-3' and B1R2: 5'-GGCGACCAATCTGCGAATACACC-3'. PCR product of 191 and 134 bp were obtained from first and second round of PCR reaction, respectively. PCR reactions $(25 \mu \mathrm{l})$ included $2.5 \mu$ l DNA, $12.5 \mu$ l commercial premix PPP master mix, 0.1 $\mu l$ each primers $(0.1 \mathrm{mM})$ and $9.8 \mu \mathrm{l}$ nuclease-free water. The amplification conditions included a 5 min of initial denaturation at $94^{\circ} \mathrm{C}$, followed by 35 cycles of $94^{\circ} \mathrm{C}$ for $10 \mathrm{~s}$ (denaturation), $57^{\circ} \mathrm{C}$ for $10 \mathrm{~s}$ (annealing), $72^{\circ} \mathrm{C}$ for $30 \mathrm{~s}$ (extension), and a final extension step at $72^{\circ} \mathrm{C}$ for $5 \mathrm{~min}$. The amplification condition for the secondary PCR was identical to the primary PCR, except that the annealing temperature was $63^{\circ} \mathrm{C}(21)$. Positive (GT1 strain) and negative (ultrapure $\mathrm{H}_{2} \mathrm{O}$ ) controls were included in each assay.
The N. caninum NC-5 gene (328 bp) was amplified using PCR as previously described $(26,27)$, and by using reaction conditions and primers (Np21: GGGTGTGCGTCCAATCCTGTAAC; NP6: CTCGCCAGTCAACCTACGTCTTCT) described previously (28). The PCR amplification reaction included $3 \mu 1$ of total DNA, $12.5 \mu \mathrm{l}$ of commercial premix PPP master mix, $0.1 \mu \mathrm{l}$ of each PCR prime $(0.1 \mathrm{mM})$ and the remaining $25 \mu \mathrm{l}$ reaction volume was topped up with nuclease-free water. The amplification conditions included $5 \mathrm{~min}$ initial denaturation at $94^{\circ} \mathrm{C}$, followed by 40 cycles of amplification $\left(40 \mathrm{~s}\right.$ at $94^{\circ} \mathrm{C}, 40 \mathrm{~s}$ at $94^{\circ} \mathrm{C}, 40 \mathrm{~s}$ at $72^{\circ} \mathrm{C}$ and a final extension step at $72^{\circ} \mathrm{C}$ for $10 \mathrm{~min}$. Positive $(N$. caninum NC-1 strain) and negative (ultrapure $\mathrm{H}_{2} \mathrm{O}$ ) controls were included in each assay.

Each PCR product was examined on agarose gel (1\%) electrophoresis to verify that they presented the expected bands of the target genes. The positive PCR products for the NC-5 gene were submitted to the Sangon Biotech Company (Shanghai, China) for DNA sequencing.

\section{Genetic Characterization of T. gondii}

The B1 gene-positive samples were genotyped at 10 genetic markers (SAG1, SAG2 (5'+3' SAG2, alter. SAG2), SAG3, BTUB, GRA6, c22-8, c29-2, L358, PK1, and Apico) using the multi-locus PCR-RFLP analysis as previously described (29) (Table 2). Eight reference T. gondii strains (GT1, PTG, CTG, MAS, TgCgCa1, TgCatBr5, TgCatBr64, and TgRsCr1) were included as controls as reported in previous studies $(33,34)$. The genotype was determined by comparing its multilocus pattern to the pattern of all genotypes present in ToxoDB (http://toxodb.org/toxo/) (35).

\section{Statistical Analysis}

The data were analyzed using SPSS 20.0 (IBM, Chicago, IL, USA). Multivariable mixed-effects logistic regression model was used to determine the relationship between the prevalence of $T$. gondii and various factors related to the ducks examined in the study, including the geographic region, the season of collection, age, and sex. Probability $(P)$ value $<0.05$ was considered as statistical significance.

\section{RESULTS}

In this study, the overall prevalence of T. gondii in domestic ducks in Hunan province was $7.7 \%$ (95\% CI: 5.5-9.9) (45/588). The prevalence of T. gondii infection in domestic ducks was $2.6 \%, 9.2$, 9.3, 11.5, and 4.6\% in Eastern, Southern, Central, Western, and Northern Hunan, respectively. However, there was no significant statistical difference in domestic ducks from different regions $(P>0.05)$ in Hunan province compared to Western region $(P<0.05)$ (Table 1). The prevalence of T. gondii infection in different seasons is shown in Table 1. The highest prevalence was found in Autumn (11.5\%; 95\%CI:6.6-16.4), followed by Summer (9.7\%; 95\%CI: 5.4-14.0) and Spring (2.4\%; 95\%CI: 05.7), and these differences were statistically significant $(P<0.05)$ compared to Winter. The prevalence of T. gondii in domestic ducks of $1<$ years $\leq 2$ (8.6\%; 95\% CI: $6.1-11.1)$ was higher than ducks of $0<$ year $\leq 1 \quad(2.2 \%$; 95\% CI: $0-5.2)$ (Table 1), and these differences were statistically significant $(P<0.05)$. Logistic 
TABLE 1 | Prevalence and risk factors for Toxoplasma gondii infection in domestic ducks in Hunan province, China.

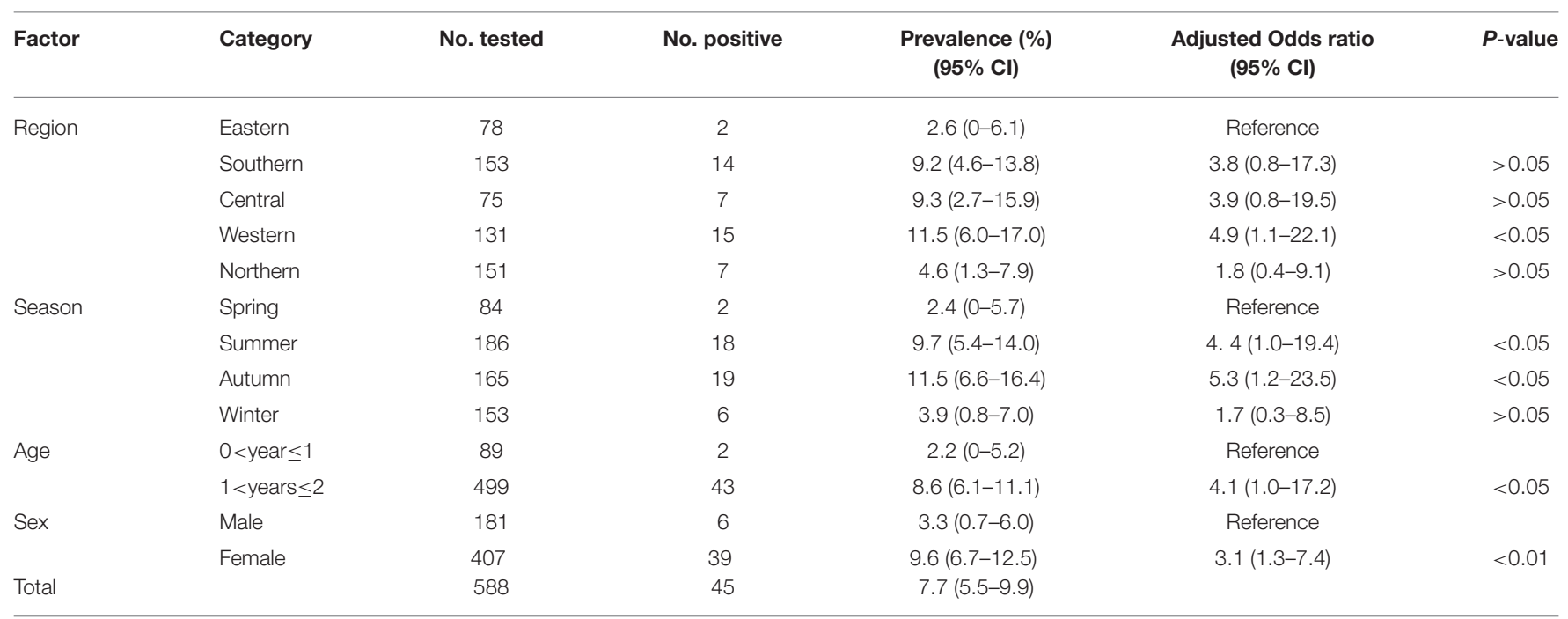

regression analysis showed that ducks $1<$ years $\leq 2$ of age (OR: 4.1 ; 95\% CI: 1.0-17.2) had four times higher risk of being positive compared with ducks $\leq 1$ year old. As is shown in Table 1 , female ducks $(9.6 \%, 95 \%$ CI: 6.7-12.5) had a higher prevalence than male ducks $(3.3 \%, 95 \%$ CI: 0.7-6.0), and these differences were statistically significant $(P<0.01)$. Logistic regression analysis showed that female ducks (OR: 3.1; 95\% CI: 1.3-7.4) had three times higher risk of acquiring $T$. gondii infection compared with male ducks. In the present study, only one brain sample was genotyped at all loci, which was identified as genotype ToxoDB\#9 (Table 2).

Two (0.3\%; 95\% CI: $0-0.7)$ of the 588 examined brain samples were positive for $N$. caninum Nc-5 gene. The sequences of the amplicons of both samples were deposited in GenBank (GenBank accession nos. MW194292 and MW194293). The Nc-5 gene sequences of $N$. caninum had $99 \%$ similarity to $N$. caninum sequence published previously (GenBank accession no. KU253799).

\section{DISCUSSION}

The prevalence $(7.7 \%)$ of $T$. gondii in ducks in present study was higher than that reported in doves (Zenaida macroura) in American country (1\%) (36); pigeon (Columba livia) in Iran (6.9\%) (37); wild ducks in the Czech Republic (38); and poultry in Shandong (7.37\%) (19). However, this prevalence was significantly lower than that detected in starlings (Sturnus vulgaris) (12.8\%); chickens (Gallus domesticus) (15.5\%) and sparrows (Passer domesticus) (26.5\%) in Iran (37) and sparrows (Passer domesticus) in Brazil (17.5\%) (39). These differences might be related to different avian species or different husbandry practices.

The results showed that ducks $1<$ years $\leq 2$ of age (OR: 4.1; 95\% CI: 1.0-17.2) had four times higher risk of being positive compared with ducks $\leq 1$ year-old, indicating that age may be a risk factor for $T$. gondii infection, in agreement with previous studies (40-43). Age is widely considered as a risk factor for high infection rates of $T$. gondii $(44,45)$. This might be attributed to increased frequency of exposure to the infectious $T$. gondii oocysts or the cumulative effect of the time period during which an animal can be exposed to the parasite $(43,46)$. The present study also showed that female ducks (OR: 3.1; 95\% CI: 1.37.4) had three times higher risk of acquiring T. gondii infection compared with male ducks, suggesting that female ducks are more susceptible to T. gondii than male ducks (47).

In the present study, only one brain sample showed complete genotype at all loci, which was identified as genotype ToxoDB\#9 (Table 2), which is consistent with that reported in ducks in a previous study in China (19). In addition, the remaining $44 \mathrm{B1-}$ positive samples were amplified at only 3-5 loci, so have limited significance to reveal the level of genetic variation of $T$. gondii. To date, although different genotypes of $T$. gondii have been reported in domestic avian species worldwide (e.g., ToxoDB\#2, 9, $10,26,53,114,225,227,278,281,282)(19,48-52)$, ToxoDB\#9 is the prominent genotype in domestic poultry in China, and has been also frequently reported in other animals in China (53). A previous study (19) indicated that only one genotype (ToxoDB\#9) was identified from domestic ducks, suggesting that the genetic variation of $T$. gondii may be relatively low in domestic ducks in China. However, further investigations including more domestic duck samples from other provinces of China are required to ascertain the full extent of T. gondii genotypes in domestic ducks.

Although previous studies showed that $N$. caninum DNA has been detected in domestic and wild poultry $(20,54)$, it was not detected in domestic ducks. The present study revealed a low molecular prevalence $(0.3 \%)$ of $N$. caninum in domestic ducks, which is significantly lower than that reported in wild waterfowl (28.6\%) (20) and chickens (4\%) (54). Differences in N. caninum prevalence are likely attributed to differences in climates, husbandry practices, detection methods, or geographical origins. Our finding provided further evidence that domestic ducks are 


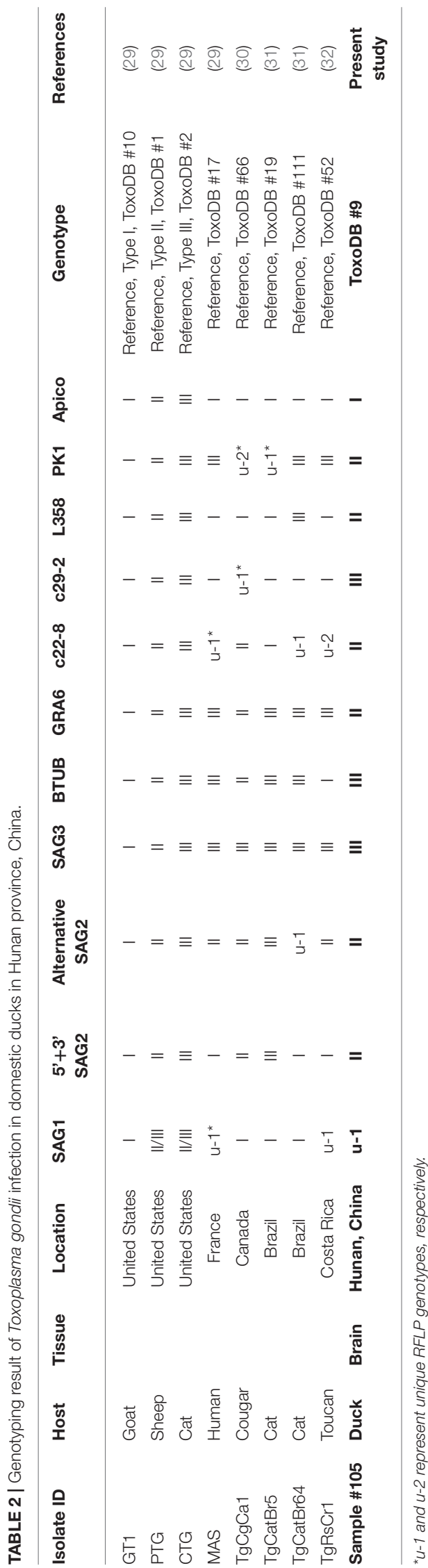

natural intermediate hosts N. caninum. Previous studies (5557) have shown that poultry get infected with $T$. gondii through ingestion of sporulated oocysts from a contaminated soil. So, it is possible that domestic ducks become infected with $N$. caninum via the same route.

Humans become infected with $T$. gondii mainly via ingestion of raw or undercooked meat of infected animals (58). The present and previous (19) studies revealed the presence of $T$. gondii infection in domestic ducks in China, highlighting the potential threat to human health. According to the Ministry of Agriculture and Rural Affairs of China, 9,444,400 metric tons (about $70 \%$ of the global total) (59) of duck meat was produced and consumed in China in 2019. Duck meat including roast, spicy and dried duck meat is very popular among most Chinese. More importantly, pregnant women are encouraged to consume duck products (including duck blood) due to cultural habits. The risk of $T$. gondii infection in humans greatly increases by eating undercooked infected meat or meat products obtained from ducks. Therefore, adequate cooking of potentially infected duck meat is the safest way to ensure that tissue cysts are deactivated, thereby preventing infection. The results of the present study should assist the duck meat industry and local regulatory agencies to optimize interventions to improve the safety of duck products.

\section{CONCLUSION}

The present study provided new data on the prevalence and risk factors of $T$. gondii infection in domestic ducks in Hunan province, China. To our knowledge, this is the first study focusing on $N$. caninum in domestic ducks in China. Future studies should consider studying histopathological changes and viability assessment of the parasites present in the duck tissues. Our findings provide a baseline for future surveillance and control of these parasites in ducks in China and reaffirm the importance of a "One Health" approach to dealing with toxoplasmosis.

\section{DATA AVAILABILITY STATEMENT}

The datasets presented in this study can be found in online repositories. The names of the repository/repositories and accession number(s) can be found at: GenBank and accessions MW194292 and MW194293.

\section{ETHICS STATEMENT}

The study was approved by the Ethics Committee of Hunan Agricultural University (No. 43321503).

\section{AUTHOR CONTRIBUTIONS}

G-HL conceived and designed the study and critically revised the manuscript. Q-YL performed the experiments, analyzed the data, and drafted the manuscript. H-LZ and W-HY helped in the study design. All authors read and approved the final manuscript. 


\section{FUNDING}

This work was provided in part by the Training Program for Excellent Young Innovators of Changsha (Grant No. KH2002001), and the Planned Programme of Hunan Province Science and Technology Innovation (Grant no. 2018RS3085).

\section{REFERENCES}

1. Frenkel JK, Smith DD. Determination of the genera of cyst-forming coccidia. Parasitol Res. (2003) 91:384-9. doi: 10.1007/s00436-003-0969-4

2. Tenter AM, Heckeroth AR, Weiss LM. Toxoplasma gondii: from animals to humans. Int $J$ Parasitol. (2000) 30:121758. doi: 10.1016/S0020-7519(00)00124-7

3. Sukthana Y. Toxoplasmosis: beyond animals to humans. Trends Parasitol. (2006) 22:137-42. doi: 10.1016/j.pt.2006.01.007

4. Pan M, Lyu C, Zhao J, Shen B. Sixty years (1957-2017) of research on toxoplasmosis in China-an overview. Front Microbiol. (2017) 8:1825. doi: $10.3389 /$ fmicb. 2017.01825

5. Elsheikha HM. Congenital toxoplasmosis: priorities for further health promotion action. Public Health. (2008) 122:33553. doi: 10.1016/j.puhe.2007.08.009

6. Switaj K, Master A, Skrzypczak M, Zaborowski P. Recent trends in molecular diagnostics for Toxoplasma gondii infections. Clin Microbiol Infect. (2005) 11:170-6. doi: 10.1111/j.1469-0691.2004.01073.x

7. Trees A, Davison H, Innes E, Wastling J. Towards evaluating the economic impact of bovine neosporosis. Int J Parasitol. (1999) 29:1195200. doi: 10.1016/S0020-7519(99)00093-4

8. Dubey JP, Schares G, Ortega-Mora LM. Epidemiology and control of neosporosis and Neospora caninum. Clin Microbiol Rev. (2007) 20:32367. doi: 10.1128/CMR.00031-06

9. Tranas J, Heinzen RA, Weiss LM, McAllister MM. Serological evidence of human infection with the protozoan Neospora caninum. Clin Diagn Lab Immunol. (1999) 6:765-7. doi: 10.1128/CDLI.6.5.765-767.1999

10. Lobato J, Silva DA, Mineo TW, Amaral JD, Segundo GR, Costa-Cruz JM, et al. Detection of immunoglobulin G antibodies to Neospora caninum in humans: high seropositivity rates in patients who are infected by human immunodeficiency virus or have neurological disorders. Clin Vaccine Immunol. (2006) 13:84-9. doi: 10.1128/CVI.13.1.84-89.2006

11. Ibrahim HM, Huang P, Salem TA, Talaat RM, Nasr MI, Xuan X, et al. Short report: prevalence of Neospora caninum and Toxoplasma gondii antibodies in northern Egypt. Am J Trop Med Hyg. (2009) 80:26367. doi: 10.4269/ajtmh.2009.80.263

12. Moskwa B, Kornacka A, Cybulska A, Cabaj W, Reiterova K, Bogdaszewski M, et al. Seroprevalence of Toxoplasma gondii and Neospora caninum infection in sheep, goats and fallow deer farmed on the same area. J Anim Sci. (2018) 96:2468-73. doi: 10.1093/jas/sky122

13. Dubey JP. Toxoplasmosis of Animals and Humans. Boca Raton: FL CRC Press (2010).

14. Dubey JP, Hemphill A, Schares G, Calero-Bernal R. Neosporosis in Animals. Boca Raton, FL: CRC Press, Taylor \&Francis Group. (2017) doi: 10.1201/9781315152561

15. Montoya JG, Liesenfeld O. Toxoplasmosis. Lancet. (2004) 363:196576. doi: 10.1016/S0140-6736(04)16412-X

16. Pagmadulam B, Myagmarsuren P, Fereig RM, Igarashi M, Yokoyama N, Battsetseg B, et al. Seroprevalence of Toxoplasma gondii and Neospora caninum infections in cattle in Mongolia. Vet Parasitol Reg Stud Rep. (2018) 14:11-7. doi: 10.1016/j.vprsr.2018.08.001

17. Wang D, Liu Y, Jiang T, Zhang G, Yuan G, He J, et al. Seroprevalence and genotypes of Toxoplasma gondii isolated from pigs intended for human consumption in Liaoning province, northeastern China. Parasites Vectors. (2016) 9:248. doi: 10.1186/s13071-016-1525-2

18. Wang L, Chen H, Liu D, Huo X, Gao J, Song X, et al. Genotypes and mouse virulence of Toxoplasma gondii isolates from animals and Humans in China. PLoS ONE. (2013) 8:e53483. doi: 10.1371/journal.pone.0053483

\section{ACKNOWLEDGMENTS}

The authors would like to thank professor Hany M. Elsheikha, University of Nottingham for constructive comments and valuable insight, which improved the clarity of the manuscript.

19. Zou Y, Nie LB, Zhang NZ, Zou FC, Zhu XQ, Cong W. First genetic characterization of Toxoplasma gondii infection in poultry meat intended for human consumption in eastern China. Infect Genet Evol. (2017) 55:1724. doi: 10.1016/j.meegid.2017.08.022

20. Rocchigiani G, Poli A, Nardoni S, Papini R, Mancianti F. Neospora caninum in wild waterfowl: occurrence of parasite DNA and low antibody titers. $J$ Parasitol. (2017) 103:142-5. doi: 10.1645/16-34

21. Hill DE, Chirukandoth S, Dubey JP, Lunney JK, Gamble HR. Comparison of detection methods for Toxoplasma gondii in naturally and experimentally infected swine. Vet Parasitol. (2006) 141:9-17. doi: 10.1016/j.vetpar.2006.05.008

22. Gui BZ, Zheng WB, Zou Y, Lv QY, Liu MT, Li F, et al. Molecular detection and genotyping of Toxoplasma gondii in pigs for human consumption in Hunan Province, China. Foodborne Pathog Dis. (2018) 15:809-13. doi: 10.1089/fpd.2018.2517

23. Ai K, Huang CQ, Guo JJ, Cong H, He SY, Zhou CX, et al. Molecular detection of Toxoplasma gondii in the slaughter sheep and goats from Shandong Province, eastern China. Vector-Borne Zoonot. (2019) 20:1936. doi: 10.1089/vbz.2019.2488

24. Mahami-Oskouei M, Moradi M, Fallah E, Hamidi F, Asl R, Akbari N, et al. Molecular detection and genotyping of Toxoplasma gondii in chicken, beef, and lamb meat consumed in northwestern Iran. Iran J Parasitol. (2017) 12:3845.

25. Burg JL, Grover CM, Pouletty P, Boothroyd JC. Direct and sensitive detection of a pathogenic protozoan, Toxoplasma gondii, by polymerase chain reaction. J Clin Microbiol. (1989) 27:1787-92. doi: 10.1128/JCM.27.8.1787-1792.1989

26. Yamage $\mathrm{M}$, Flechtner O, Gottstein B. Neospora caninum: specific oligonucleotide primers for the detection of brain "cyst" DNA of experimentally infected nude mice by the Polymerase Chain Reaction (PCR). J Parasitol. (1996) 82:272. doi: 10.2307/3284160

27. Lin MH, Chen TC, Kuo TT, Tseng CC, Tseng CP. Real-time PCR for quantitative detection of Toxoplasma gondii. J Clin Microbiol. (2000) 38:41215. doi: 10.1128/JCM.38.11.4121-4125.2000

28. Liu MT, Lv QY, Jiang WX, Li J, Gui BZ, Zheng WB, et al. Molecular detection of Neospora caninum from naturally infected four passeriforme birds in China. Acta Trop. (2019) 197:105044. doi: 10.1016/j.actatropica.2019.105044

29. Su C, Shwab EK, Zhou P, Zhu XQ, Dubey JP. Moving towards an integrated approach to molecular detection and identification of Toxoplasma gondii. Parasitology. (2010) 137:1-1. doi: 10.1017/S00311820099 91065

30. Dubey JP, Quirk T, Pitt JA, Sundar N, Velmurugan GV, Kwok OCH, et al. Isolation and genetic characterization of Toxoplasma gondii from raccoons (Procyon lotor), cats (Felis domesticus), striped skunk (Mephitis mephitis), black bear (Ursus americanus), and cougar (Puma concolor) from Canada. J Parasitol. (2008) 94:42-5. doi: 10.1645/GE-1349.1

31. Pena HFJ, Gennari SM, Dubey JP, Su C. Population structure and mousevirulence of Toxoplasma gondii in Brazil. Int J Parasitol. (2008) 38:5619. doi: 10.1016/j.ijpara.2007.09.004

32. Dubey JP, Velmurugan GV, Morales JA, Arguedas R, Su C. Isolation of Toxoplasma gondii from the keel-billed toucan (Ramphastos sulfuratus) from Costa Rica. J Parasitol. (2009) 95:467-8. doi: 10.1645/GE-1846.1

33. Su C, Zhang X, Dubey JP. Genotyping of Toxoplasma gondii by multilocus PCR-RFLP markers: a high resolution and simple method for identification of parasites. Int J Parasitol. (2006) 36:841-8. doi: 10.1016/j.ijpara.2006.03.003

34. Zheng WB, Gui BZ, Long HB, Chen YW, Zhu XQ, Wang SL, et al. Molecular detection and genotyping of Toxoplasma gondii in Edward's longtailed rats (Leopoldamys edwardsi). Foodborne Pathog Dis. (2019) 16:53942. doi: 10.1089/fpd.2018.2605 
35. Fernández-Escobar M, Calero-Bernal R, Regidor-Cerrillo J, Vallejo R, Benavides J, Collantes-Fernández E, et al. Isolation, genotyping, and mouse virulence characterization of Toxoplasma gondii from free ranging Iberian pigs. Front Vet Sci. (2020) 7:604782. doi: 10.3389/fvets.2020.604782

36. Ammar S, Purple K, Gerhold R. Toxoplasma gondii prevalence in hunter-killed mourning doves (Zenaida macroura) and rock pigeons (Columba livia) from East Tennessee. J Wildlife Dis. (2020) 56:479481. doi: 10.7589/2019-06-155

37. Khademvatan S, Saki J, Yousefi E, Abdizadeh R. Detection and genotyping of Toxoplasma gondii strains isolated from birds in the southwest of Iran. Brit Poultry Sci. (2013) 54:76-80. doi: 10.1080/00071668.2013.7 63899

38. Skorpikova L, Reslova N, Lorencova A, Plhal R, Drimaj J, Kamler J, et al. Molecular detection of Toxoplasma gondii in feathered game intended for human consumption in the Czech Republic. Int J Food Microbiol. (2018) 286:75-9. doi: 10.1016/j.ijfoodmicro.2018.07.019

39. Gondim LSQ, Abe-Sandes K, Uzêda RS, Silva MSA, Santos SL, Mota RA, et al. Toxoplasma gondii and Neospora caninum in sparrows (Passer domesticus) in the Northeast of Brazil. Vet Parasitol. (2010) 168:1214. doi: 10.1016/j.vetpar.2009.09.055

40. Cabezón O, García-Bocanegra I, Molina-López R, Marco I, Blanco JM, Höfle U, et al. Seropositivity and risk factors associated with Toxoplasma gondii infection in wild birds from Spain. PLoS ONE. (2011) 6:e29549. doi: 10.1371/journal.pone.0029549

41. Alvarado-Esquivel C, González-Salazar AM, Alvarado-Esquivel D, OntiverosVázquez F, Vitela-Corrales J, Villena I, et al. Seroprevalence of Toxoplasma gondii infection in chickens in Durango State, Mexico. J Parasitol. (2012) 98:431-432. doi: 10.1645/GE-2979.1

42. Li MH, Yang BT, Yin ZW, Wang W, Zhao Q, Jiang J. A seroepidemiological survey of Toxoplasma gondii and Chlamydia infection in chickens, ducks, and geese in Jilin province, northeastern China. Vector-Borne Zoonot. (2020) 20:825-30. doi: 10.1089/vbz.2020.2614

43. Rong G, Zhou HL, Hou GY, Zhao JM, Xu TS, Guan S. Seroprevalence, risk factors and genotyping of Toxoplasma gondii in domestic geese (Anser domestica) in tropical China. Parasite Vector. (2014) 7:459. doi: 10.1186/PREACCEPT-1506796653138778

44. Must K, Lassen B, Jokelainen P. Seroprevalence of and risk factors for Toxoplasma gondii infection in cats in Estonia. Vector Borne Zoonotic Dis. (2015) 15:597-601. doi: 10.1089/vbz.2015.1809

45. Yan X, Han W, Wang Y, Zhang, H, Gao Z. Seroprevalence of Toxoplasma gondii infection in sheep in Inner Mongolia Province, China. Parasite. (2020) 27:11. doi: 10.1051/parasite/2020008

46. Lücht M, Stagegaard J, Conraths FJ, Schares G. Toxoplasma gondii in small exotic felids from zoos in Europe and the Middle East: serological prevalence and risk factors. Parasites Vectors. (2019) 12:449. doi: 10.1186/s13071-019-3706-2

47. Khan MB, Khan S, Rafiq K, Khan SN, Attaullah S, Ali I. Molecular identification of Toxoplasma gondii in domesticated and broiler chickens (Gallus domesticus) that possibly augment the pool of human toxoplasmosis. PLoS ONE. (2020) 15:e0232026. doi: 10.1371/journal.pone.0 232026

48. Dubey JP, Graham DH, Dahl E, Hilali M, El-Ghaysh A, Sreekumar $\mathrm{C}$, et al. Isolation and molecular characterization of Toxoplasma gondii from chickens and ducks from Egypt. Vet Parasitol. (2003) 114:8995. doi: 10.1016/S0304-4017(03)00133-X

49. Wang L, Cheng HW, Huang KQ, Xu YH, Li YN, Du J, et al. Toxoplasma gondii prevalence in food animals and rodents in different regions of China: isolation, genotyping and mouse pathogenicity. Parasit Vectors. (2013) 6:273. doi: 10.1186/1756-3305-6-273

50. Pena HFJ, Alves BF, Soares HS, Oliveira S, Ferreira MN, Bricarello PA, et al. Free-range chickens from Santa Catarina state, southern Brazil, as asymptomatic intermediate hosts for Toxoplasma gondii clonal type I and typical Brazilian genotypes. Vet Parasitol Reg Stu Reports. (2018) 13:5559. doi: 10.1016/j.vprsr.2018.04.001

51. Hamilton CM, Robins R, Thomas R, Oura C, Oliveira S, Villena I, et al. Prevalence and genetic diversity of Toxoplasma gondii in freeranging chickens from the Caribbean. Acta Parasitol. (2019) 64:73844. doi: 10.2478/s11686-019-00071-7

52. Ribeiro-Andrade M, de-Crasto-Souza-Carvalho J, Amorim-da-Silva R, daConceição- Carvalho M, Nascimento-Porto WJ, Mota RA. Inter- and intragenotype differences in induced cystogenesis of recombinant strains of Toxoplasma gondii isolated from chicken and pigs. Exp Parasitol. (2019) 207:107775. doi: 10.1016/j.exppara.2019.107775

53. Dong H, Su R, Lu Y, Wang M, Liu J, Jian F, et al. Prevalence, risk factors, and genotypes of Toxoplasma gondii in food animals and humans (2000-2017) from China. Front Microbiol. (2018) 9:2108. doi: 10.3389/fmicb.2018.02108

54. Romero DG, Sánchez GFD, Morales SE. Neospora caninum in free-range chickens of Central Mexico. Vet Parasitol Reg Stud Reports. (2016) 5:313. doi: 10.1016/j.vprsr.2016.08.006

55. Ruiz A, Frenkel JK. Intermediate and transport hosts of Toxoplasma gondii in Costa Rica. Am J Trop Med Hyg. (1980) 29:1161-6. doi: 10.4269/ajtmh.1980.29.1161

56. Yan C, Yue CL, Yuan ZG, He Y, Yin CC, Lin RQ, et al. Toxoplasma gondii infection in domestic ducks, free-range and caged chickens in southern China. Vet Parasitol. (2009) 165:337-40. doi: 10.1016/j.vetpar.2009.07.015

57. Ibrahim HM, Osman GY, Mohamed AH, Al-Selwi AGM, Nishikawa Y, AbdelGhaffar F. Toxoplasma gondii: Prevalence of natural infection in pigeons and ducks from middle and upper Egypt using serological, histopathological, and immunohistochemical diagnostic methods. Vet Parasitol Reg Stu Reports. (2018) 13:45-9. doi: 10.1016/j.vprsr.2018.04.002

58. Hill D, Dubey JP. Toxoplasma gondii: transmission, diagnosis and prevention. Clin Microbiol Infect. (2002) 8:634-40. doi: 10.1046/j.1469-0691.2002.00485.x

59. Zeng T, Chen L, Du X, Lai SJ, Huang SP, Liu YL, et al. Association analysis between feed efficiency studies and expression of hypothalamic neuropeptide genes in laying ducks. Anim Genet. (2016) 47:606-9. doi: 10.1111/age.12457

Conflict of Interest: The authors declare that the research was conducted in the absence of any commercial or financial relationships that could be construed as a potential conflict of interest.

Copyright (C) $2021 \mathrm{Lv}$, Zheng, Yang and Liu. This is an open-access article distributed under the terms of the Creative Commons Attribution License (CC BY). The use, distribution or reproduction in other forums is permitted, provided the original author(s) and the copyright owner(s) are credited and that the original publication in this journal is cited, in accordance with accepted academic practice. No use, distribution or reproduction is permitted which does not comply with these terms. 